\title{
Maximum penalized likelihood estimation for a stress-strength reliability model using complete and incomplete data
}

\author{
Marwa Khalil Hassan ${ }^{1, a, b}$ \\ ${ }^{a}$ Department of Mathematics, Ain Shams University, Egypt; \\ ${ }^{b}$ Department of Mathematics, Imam Abdulrahman Bin Faisal University, Saudi Arabia
}

\begin{abstract}
The two parameter negative exponential distribution has many practical applications in queuing theory such as the service times of agents in system, the time it takes before your next telephone call, the time until a radioactive practical decays, the distance between mutations on a DNA strand, and the extreme values of annual snowfall or rainfall; consequently, has many applications in reliability systems. This paper considers an estimation problem of stress-strength model with two parameter negative parameter exponential distribution. We introduce a maximum penalized likelihood method, Bayes estimator using Lindley approximation to estimate stress-strength model and compare the proposed estimators with regular maximum likelihood estimator for complete data. We also introduce a maximum penalized likelihood method, Bayes estimator using a Markov chain Mote Carlo technique for incomplete data. A Monte Carlo simulation study is performed to compare stress-strength model estimates. Real data is used as a practical application of the proposed model.

Keywords: maximum penalized likelihood estimator, Lindley approximation, progressive type II censored data, Bayes estimator, simulation, Markov chain Mote Carlo technique, two parameter negative exponential distribution
\end{abstract}

\section{Introduction}

Let $X$ be a two parameter negative exponential distribution with scale parameter $\lambda$ measuring the failure rate and location parameter $\mu$. Then the probability density function and cumulative distribution function of $X$ are

$$
f(x ; \mu, \lambda)=\frac{1}{\lambda} \exp \left[-\frac{(x-\mu)}{\lambda}\right] I(x>\mu), \quad x>\mu, \mu \geq 0, \lambda>0
$$

and

$$
F(x ; \mu, \lambda)=1-\exp \left[-\frac{(x-\mu)}{\lambda}\right] I(x>\mu), \quad x>\mu,
$$

\footnotetext{
${ }^{1}$ Department of Mathematics, Faculty of Education, Ain Shams University, Cairo, Egypt and Department of Mathematics, College of Science, Imam Abdulrahman Bin Faisal University, Saudi Arabia. E-mail: marwa_khali12006@hotmail.com
}

Published 31 July 2018 / journal homepage: http://csam.or.kr

(C) 2018 The Korean Statistical Society, and Korean International Statistical Society. All rights reserved. 


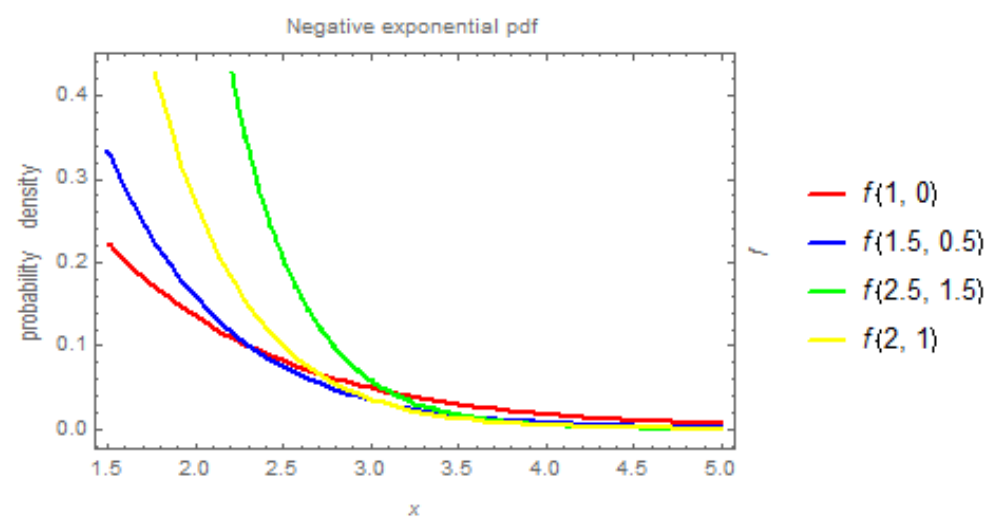

Figure 1: Two parameter negative exponential distribution.

where $I(\cdot)$ is the indicator function of $(\cdot)$ that is $I(x>\mu)=1$ for all $x>\mu$. Figure 1 shows the graph of two parameter negative exponential distribution for different values of $\mu$ and $\lambda$, we then observe that the two parameter negative exponential distribution is a positively skewed distribution.

The two parameter negative exponential distribution plays an important role in modeling in many fields such as queuing theory, physics, biology, hydrology, reliability, and reliability engineering. In this paper, we examine the problem of estimating the reliability parameter i.e., $R=P[Y<X]$. The reliability parameter is referred to the stress-strength model included in quality control, engineering statistics, and other fields. In reliability context, the stress-strength model describes the life of component which has a random strength variable $X$ and is subjected to random variable stress $Y$. The system fails any time the stress is greater than the strength. The estimation of stress-strength model when $X$ and $Y$ are random variables having a specified distribution discussed by many authors starting from Birnbaum (1956), Basu (1964), Downtown (1973), Tong (1974, 1977), Beg (1980), Iwase (1987), McCool (1991). Recently, Ali et al. (2012), Greco and Ventura (2011), Rezaei et al. (2010), Wong (2012), Shahsanaei and Daneshkhah (2013), Hussian (2013), Al-Mutairi et al. (2013), Ghitany et al. (2015), Najarzadegan et al. (2016). The estimator of reliability parameter when $X$ and $Y$ are independent exponential random variables discussed by several authors such as Enis and Geisser (1971), Kelley et al. (1976), Sathe and Shah (1981). The pervious work of reliability parameter when $X$ and $Y$ are independent two parameter negative exponential random variables discussed by Pal et al. (2005) derived the distribution of UMVUE of the reliability parameter; subsequently, Krishnamoorthy et al. (2007) studied the problem of hypothesis testing and interval estimation of the reliability parameter.

In this paper, we introduce a maximum penalized likelihood estimator of a reliability parameter when $X$ and $Y$ are independent two parameter negative exponential random variables as well as introduce the Bayes estimator of it using Lindley approximation. This paper organized as: the stress-strength parameter when $X$ and $Y$ are independent two parameter negative exponential random variables with parameters $\left(\lambda_{1}, \mu_{1}\right)$ and $\left(\lambda_{2}, \mu_{2}\right)$ respectively is determined in Section 2 . We present maximum penalized likelihood estimator of the reliability parameter and compare it by the maximum likelihood estimator also the Bayes estimator of the reliability parameter using Lindley approximation introduced for complete data in Section 3. We present the maximum penalized likelihood estimator of the reliability parameter and the Bayes estimator of the reliability parameter using a Markov chain Monte Carlo technique (MCMC) introduced for the incomplete data in Section 4. The performance of these estimator is assessed by simulation study in Section 5. A practical application is presented in Section 6. The conclusion of this paper introduced in Section 7. 


\section{Stress-strength model}

Let $X$ and $Y$ be independent strength and stress random variables follow two parameter negative exponential distribution with parameters $\left(\lambda_{1}, \mu_{1}\right)$ and $\left(\lambda_{2}, \mu_{2}\right)$ respectively. Then the stress-strength model $R$ define as

$$
R=P[Y<X]=\int_{\min \left(\mu_{1}, \mu_{2}\right)}^{\infty} F_{Y}(x) f(x) d x .
$$

For all cases of $\left(\mu_{1}, \mu_{2}\right)$ we obtain the formula of $R$ as:

- If $\mu_{1}=\mu_{2}$, then

$$
R=\frac{\lambda_{1}}{\lambda_{1}+\lambda_{2}}
$$

- If $\mu_{1} \neq \mu_{2}$, then

$$
R=\left[1-\frac{\lambda_{2}}{\lambda_{1}+\lambda_{2}} e^{\frac{\left(\mu_{2}-\mu_{1}\right)}{\lambda_{2}}}\right] I\left(\mu_{1}>\mu_{2}\right)+\left[\frac{\lambda_{1}}{\lambda_{1}+\lambda_{2}} e^{\frac{\left(\mu_{1}-\mu_{2}\right)}{\lambda_{1}}}\right] I\left(\mu_{1}<\mu_{2}\right),
$$

where $I(\cdot)$ is the indicator function.

In the next sections, we will obtain the different estimators of $R$.

\section{Different estimators of $\boldsymbol{R}$ for complete data}

In this section, the maximum penalized likelihood estimators of parameter $R$ are obtained and compared by its regular maximum likelihood estimator for complete data and the Bayes estimator of reliability parameter using the Lindley approximation also introduced for complete data.

\subsection{Maximum penalized likelihood estimator of $R$}

Zheng (2013) introduced the maximum penalized likelihood estimator of two parameter negative exponential distribution parameters because the regular maximum likelihood estimator of distribution parameters were biased. In this subsection, we use the Zheng (2013) approach to find the maximum penalized estimator of $R$ using the following algorithm.

Algorithm 1. Find maximum penalized likelihood estimator of $R$ says $R^{*}$ using the invariance prop$\overline{\text { erty as follows }}$

- Case 1: $\mu_{1}=\mu_{2}$, then

$$
\hat{R}^{*}=\frac{\hat{\lambda}_{1}^{*}}{\hat{\lambda}_{1}^{*}+\hat{\lambda}_{2}^{*}}
$$

- Case 2: $\mu_{1} \neq \mu_{2}$, then

$$
\hat{R}^{*}=\left[1-\frac{\hat{\lambda}_{2}^{*}}{\hat{\lambda}_{1}^{*}+\hat{\lambda}_{2}^{*}} e^{\frac{\hat{\mu}_{2}^{*}-\hat{\mu}_{1}^{*}}{\hat{\mu}_{2}^{*}}}\right] I\left(\hat{\mu}_{1}^{*}>\hat{\mu}_{2}^{*}\right)+\left[\frac{\hat{\lambda}_{1}^{*}}{\hat{\lambda}_{1}^{*}+\hat{\lambda}_{2}^{*}} e^{\frac{\hat{\mu}_{1}^{*}-\hat{\mu}_{2}^{*}}{\hat{\lambda}_{1}^{*}}}\right] I\left(\hat{\mu}_{1}^{*}<\hat{\mu}_{2}^{*}\right),
$$


where $\lambda_{1}^{*}, \mu_{1}^{*}, \lambda_{2}^{*}, \mu_{2}^{*}$ are the maximum penalized likelihood estimator of $\lambda_{1}, \mu_{1}, \lambda_{2}, \mu_{2}$ (Zheng, 2013).

Now, we will study the distribution of $\hat{R}^{*}$ as follows case $1: \mu_{1}=\mu_{2}$, then

$$
\hat{R}^{*}=\frac{\hat{\lambda}_{1}^{*}}{\hat{\lambda}_{1}^{*}+\hat{\lambda}_{2}^{*}}=\frac{1}{1+\hat{\lambda}_{2}^{*} / \hat{\lambda}_{1}^{*}} \text {. }
$$

Suppose $u=\hat{\lambda}_{2}^{*} / \hat{\lambda}_{1}^{*}$, then from convolution rule we get the probability density function of $U$ is $f(u)=1 /\left(1+u^{2}\right), u>0$ and the moments about zero of $\hat{R}^{*}$ is given by

$$
\begin{aligned}
E\left[\hat{R}^{*}\right]^{s} & =E[1+u]^{-s} \\
& =\frac{\pi 2^{-\frac{s}{2}}}{e^{-\frac{1}{4} i \pi s}+e^{\frac{i \pi s}{4}}}+\frac{\pi 2^{-\frac{s}{2}}}{\left(e^{-\frac{1}{4} i \pi s}+e^{\frac{i \pi s}{4}}\right)\left(e^{-\frac{1}{2} i \pi s}+e^{\frac{i \pi s}{2}}\right)}+\frac{p F_{q}\left[\left\{\frac{1}{2}, 1,1\right\},\left\{1-\frac{s}{2}, \frac{3}{2}-\frac{s}{2}\right\},-1\right]}{s-1},
\end{aligned}
$$

and the mean square error (MSE) of $\hat{R}^{*}$ is given by

$$
\operatorname{MSE}\left(\hat{R}^{*}\right)=E\left[\left(\hat{R}^{*}-R\right)^{2}\right]=\frac{\lambda_{1}^{2}-(\pi-2) \lambda_{2} \lambda_{1}+\lambda_{2}^{2}}{2\left(\lambda_{1}+\lambda_{2}\right)^{2}} .
$$

But in case $2: \mu_{1} \neq \mu_{2}$, then

$$
\hat{R}^{*}=\left[1-\frac{\hat{\lambda}_{2}^{*}}{\hat{\lambda}_{1}^{*}+\hat{\lambda}_{2}^{*}} e^{\frac{\hat{\mu}_{2}^{*}-\hat{\mu}_{1}^{*}}{\hat{\lambda}_{2}^{*}}}\right] I\left(\hat{\mu}_{1}^{*}>\hat{\mu}_{2}^{*}\right)+\left[\frac{\hat{\lambda}_{1}^{*}}{\hat{\lambda}_{1}^{*}+\hat{\lambda}_{2}^{*}} e^{\frac{\hat{\mu}_{1}^{*}-\hat{\mu}_{2}^{*}}{\hat{\lambda}_{1}^{*}}}\right] I\left(\hat{\mu}_{1}^{*}<\hat{\mu}_{2}^{*}\right) .
$$

The exact distribution of $\hat{R}^{*}$ does not exist. So, we find the asymptotic distribution of $\hat{R}^{*}$ as: as $n \rightarrow \infty$ and $m \rightarrow \infty$ the $\hat{R}^{*}$ has asymptotic normal with mean $R$ and asymptotic variance $\operatorname{Var}(R)$

$$
\operatorname{Var}(R)=\sum_{j=1}^{4} \sum_{i=1}^{4} \frac{\partial R}{\partial \Theta_{i}} \frac{\partial R}{\partial \Theta_{j}} I_{i j}^{-1}
$$

where $\Theta=\left(\lambda_{1}, \mu_{1}, \lambda_{2}, \mu_{2}\right)$ and $I_{i j}^{-1}$ is the $(i, j)^{t h}$ element of the inverse of the Fisher information matrix $I_{i j}$ which is given by

$$
\begin{aligned}
& I_{i j}=\left(\begin{array}{llll}
E\left[\frac{-\partial^{2} l^{*}(\Theta)}{\partial \lambda_{1}^{2}}\right] & E\left[\frac{-\partial^{2} l^{*}(\Theta)}{\partial \lambda_{1} \partial \mu_{1}}\right] & E\left[\frac{-\partial^{2} l^{*}(\Theta)}{\partial \lambda_{1} \partial \lambda_{2}}\right] & E\left[\frac{-\partial^{2} l^{*}(\Theta)}{\partial \lambda_{1} \partial \mu_{2}}\right] \\
E\left[\frac{-\partial^{2} l^{*}(\Theta)}{\partial \mu_{1} \partial \lambda_{1}}\right] & E\left[\frac{-\partial^{2} l^{*}(\Theta)}{\partial \mu_{1}^{2}}\right] & E\left[\frac{-\partial^{2} l^{*}(\Theta)}{\partial \mu_{1} \partial \lambda_{2}}\right] & E\left[\frac{-\partial^{2} l^{*}(\Theta)}{\partial \mu_{1} \partial \mu_{2}}\right] \\
E\left[\frac{-\partial^{2} l^{*}(\Theta)}{\partial \lambda_{2} \partial \lambda_{1}}\right] & E\left[\frac{-\partial^{2} l^{*}(\Theta)}{\partial \lambda_{2} \partial \mu_{1}}\right] & E\left[\frac{-\partial^{2} l^{*}(\Theta)}{\partial \lambda_{2}^{2}}\right] & E\left[\frac{-\partial^{2} l^{*}(\Theta)}{\partial \lambda_{2} \partial \mu_{2}}\right] \\
E\left[\frac{-\partial^{2} l^{*}(\Theta)}{\partial \mu_{2} \partial \lambda_{1}}\right] & E\left[\frac{-\partial^{2} l^{*}(\Theta)}{\partial \mu_{2} \partial \mu_{1}}\right] & E\left[\frac{-\partial^{2} l^{*}(\Theta)}{\partial \mu_{2} \partial \lambda_{2}}\right] & E\left[\frac{-\partial^{2} l^{*}(\Theta)}{\partial \mu_{2}^{2}}\right]
\end{array}\right), \\
& I_{i j}^{-1}=\left(\begin{array}{llll}
\operatorname{Var}\left(\lambda_{1}^{*}\right) & \operatorname{Cov}\left(\lambda_{1}^{*}, \mu_{1}^{*}\right) & \operatorname{Cov}\left(\lambda_{1}^{*}, \lambda_{2}^{*}\right) & \operatorname{Cov}\left(\lambda_{1}^{*}, \mu_{2}^{*}\right) \\
\operatorname{Cov}\left(\mu_{1}^{*}, \lambda_{1}^{*}\right) & \operatorname{Var}\left(\mu_{1}^{*}\right) & \operatorname{Cov}\left(\mu_{1}^{*}, \lambda_{2}^{*}\right) & \operatorname{Cov}\left(\mu_{1}^{*}, \mu_{2}^{*}\right) \\
\operatorname{Cov}\left(\lambda_{2}^{*}, \lambda_{1}^{*}\right) & \operatorname{Cov}\left(\lambda_{2}^{*}, \mu_{1}^{*}\right) & \operatorname{Var}\left(\lambda_{2}^{*}\right) & \operatorname{Cov}\left(\lambda_{2}^{*}, \mu_{2}^{*}\right) \\
\operatorname{Cov}\left(\mu_{2}^{*}, \lambda_{1}^{*}\right) & \operatorname{Cov}\left(\mu_{2}^{*}, \mu_{1}^{*}\right) & \operatorname{Cov}\left(\mu_{2}^{*}, \lambda_{2}^{*}\right) & \operatorname{Var}\left(\mu_{2}^{*}\right)
\end{array}\right)
\end{aligned}
$$


and

$$
\begin{aligned}
& \operatorname{Var}\left(\lambda_{1}^{*}\right)=\frac{\lambda_{1}^{2}}{n-1}, \quad \operatorname{Var}\left(\lambda_{2}^{*}\right)=\frac{\lambda_{2}^{2}}{m-1}, \quad \operatorname{Var}\left(\mu_{1}^{*}\right)=\frac{\lambda_{1}^{2}}{n(n-1)}, \quad \operatorname{Var}\left(\mu_{2}^{*}\right)=\frac{\lambda_{2}^{2}}{m(m-1)}, \\
& \operatorname{Cov}\left(\lambda_{1}^{*}, \mu_{1}^{*}\right)=\operatorname{Cov}\left(\mu_{1}^{*}, \lambda_{1}^{*}\right)=\frac{\lambda_{1}^{2}}{n}, \quad \operatorname{Cov}\left(\lambda_{1}^{*}, \lambda_{2}^{*}\right)=\operatorname{Cov}\left(\lambda_{2}^{*}, \lambda_{1}^{*}\right)=0, \\
& \operatorname{Cov}\left(\lambda_{1}^{*}, \mu_{2}^{*}\right)=\operatorname{Cov}\left(\mu_{2}^{*}, \lambda_{1}^{*}\right)=0, \quad \operatorname{Cov}\left(\mu_{1}^{*}, \lambda_{2}^{*}\right)=\operatorname{Cov}\left(\lambda_{2}^{*}, \mu_{1}^{*}\right)=0, \\
& \operatorname{Cov}\left(\mu_{1}^{*}, \mu_{2}^{*}\right)=\operatorname{Cov}\left(\mu_{2}^{*}, \mu_{1}^{*}\right)=0, \quad \operatorname{Cov}\left(\lambda_{2}^{*}, \mu_{2}^{*}\right)=\operatorname{Cov}\left(\mu_{2}^{*}, \lambda_{2}^{*}\right)=\frac{\lambda_{2}^{2}}{m}, \\
& \frac{\partial R}{\partial \lambda_{1}}=-\frac{\lambda_{1} e^{\frac{\mu_{1}-\mu_{2}}{\lambda_{1}}}}{\left(\lambda_{1}+\lambda_{2}\right)^{2}}+\frac{e^{\frac{\mu_{1}-\mu_{2}}{\lambda_{1}}}}{\lambda_{1}+\lambda_{2}}-\frac{\left(\mu_{1}-\mu_{2}\right) e^{\frac{\mu_{1}-\mu_{2}}{\lambda_{1}}}}{\lambda_{1}\left(\lambda_{1}+\lambda_{2}\right)} I\left(\mu_{1}<\mu_{2}\right)+\frac{\lambda_{2} e^{\frac{\mu_{2}-\mu_{1}}{\lambda_{2}}}}{\left(\lambda_{1}+\lambda_{2}\right)^{2}} I\left(\mu_{1}>\mu_{2}\right), \\
& \frac{\partial R}{\partial \lambda_{2}}=-\frac{\lambda_{1} e^{\frac{\mu_{1}-\mu_{2}}{\lambda_{1}}}}{\left(\lambda_{1}+\lambda_{2}\right)^{2}} I\left(\mu_{1}<\mu_{2}\right)+\frac{\lambda_{2} e^{\frac{\mu_{2}-\mu_{1}}{\lambda_{2}}}}{\left(\lambda_{1}+\lambda_{2}\right)^{2}}+\frac{\left(\mu_{2}-\mu_{1}\right) e^{\frac{\mu_{2}-\mu_{1}}{\lambda_{2}}}}{\lambda_{2}\left(\lambda_{1}+\lambda_{2}\right)}-\frac{e^{\frac{\mu_{2}-\mu_{1}}{\lambda_{2}}}}{\lambda_{1}+\lambda_{2}} I\left(\mu_{1}>\mu_{2}\right), \\
& \frac{\partial R}{\partial \mu_{1}}=\frac{e^{\frac{\mu_{1}-\mu_{2}}{\lambda_{1}}}}{\lambda_{1}+\lambda_{2}} I\left(\mu_{1}<\mu_{2}\right)+\frac{e^{\frac{\mu_{2}-\mu_{1}}{\lambda_{2}}}}{\lambda_{1}+\lambda_{2}} I\left(\mu_{1}>\mu_{2}\right), \\
& \frac{\partial R}{\partial \mu_{2}}=-\frac{e^{\frac{\mu_{1}-\mu_{2}}{\lambda_{1}}}}{\lambda_{1}+\lambda_{2}} I\left(\mu_{1}<\mu_{2}\right)-\frac{e^{\frac{\mu_{2}-\mu_{1}}{\lambda_{2}}}}{\lambda_{1}+\lambda_{2}} I\left(\mu_{1}>\mu_{2}\right) .
\end{aligned}
$$

Hence an asymptotic $100(1-\alpha) \%$ confidence interval for $R$ is given by

$$
R \in\left(\hat{R}^{*} \pm \frac{Z_{\frac{\alpha}{2}}}{\sqrt{n+m}} \sqrt{\widehat{\operatorname{Var}}\left(R^{*}\right)}\right)
$$

where $Z_{\alpha / 2}$ is the upper $(\alpha / 2)^{\text {th }}$ quantile of standard normal distribution and $\widehat{\operatorname{Var}}\left(R^{*}\right)$ is the value of $\operatorname{Var}\left(R^{*}\right)$ at maximum penalized likelihood estimators of parameters $\Theta=\left(\lambda_{1}, \mu_{1}, \lambda_{2}, \mu_{2}\right)$.

\subsection{Bayes estimator of $R$}

Lindley (1980) proposed on approximation technique to find the Bayes estimator of the stress-strength parameter under squared error loss function and under Jeffrey prior $(\rho)$ as follows.

\section{Algorithm 2.}

1. Find the penalized maximum likelihood estimator of $\Theta=\left(\lambda_{1}, \mu_{1}, \lambda_{2}, \mu_{2}\right)$ and $R$ says $\Theta^{*}=\left(\lambda_{1}^{*}, \mu_{1}^{*}\right.$, $\lambda_{2}^{*}, \mu_{2}^{*}$ ) and $\hat{R}^{*}$.

2. Compute the Bayes estimator of $R$ says $R^{B}$ as

$$
R^{B}=\hat{R}^{*}+\frac{1}{2}\left(\sum_{i=1}^{4} \sum_{j=1}^{4}\left(\hat{R}_{i j}^{*}+2 \hat{R}_{i}^{*} \rho_{j}\right) \sigma_{i j}\right)+\frac{1}{2}\left(\sum_{i=1}^{4} \sum_{j=1}^{4} \sum_{k=1}^{4} \sum_{l=1}^{4} L_{i j k} \sigma_{i j} \sigma_{k l} \hat{R}_{l}^{*}\right) .
$$

Note: all terms and derivatives are calculated by replacing $\Theta=\left(\lambda_{1}, \mu_{1}, \lambda_{2}, \mu_{2}\right)$ and $R$ with their 
maximum penalized likelihood estimator. Where

$$
\begin{aligned}
& R_{11}=\frac{\partial^{2} \hat{R}^{*}}{\partial \lambda_{1}^{2}}=-\frac{2 \lambda_{2} e^{\frac{\mu_{2}-\mu_{1}}{\lambda_{2}}}}{\left(\lambda_{1}+\lambda_{2}\right)^{3}} I\left[\mu_{1}>\mu_{2}\right] \\
& +\frac{e^{\frac{\mu_{1}-\mu_{2}}{\lambda_{1}}}\left(-2 \lambda_{1}^{3}\left(\lambda_{2}-\mu_{1}+\mu_{2}\right)+\lambda_{1}^{2}\left(\mu_{1}-\mu_{2}\right)\left(2 \lambda_{2}+\mu_{1}-\mu_{2}\right)+2 \lambda_{2} \lambda_{1}\left(\mu_{1}-\mu_{2}\right)^{2}+\lambda_{2}^{2}\left(\mu_{1}-\mu_{2}\right)^{2}\right)}{\lambda_{1}^{3}\left(\lambda_{1}+\lambda_{2}\right)^{3}} I\left[\mu_{1}<\mu_{2}\right], \\
& R_{12}=R_{21}=\frac{\partial^{2} \hat{R}^{*}}{\partial \lambda_{1} \partial \mu_{1}}=-\frac{e^{\frac{\mu_{2}-\mu_{1}}{\lambda_{2}}}}{\left(\lambda_{1}+\lambda_{2}\right)^{2}} I\left[\mu_{1}>\mu_{2}\right]-\frac{\left(\mu_{1}-\mu_{2}\right) e^{\frac{\mu_{1}-\mu_{2}}{\lambda_{1}}}}{\lambda_{1}^{2}\left(\lambda_{1}+\lambda_{2}\right)}-\frac{e^{\frac{\mu_{1}-\mu_{2}}{\lambda_{1}}}}{\left(\lambda_{1}+\lambda_{2}\right)^{2}} I\left[\mu_{1}<\mu_{2}\right], \\
& R_{13}=R_{31}=\frac{\partial^{2} \hat{R}^{*}}{\partial \lambda_{1} \partial \lambda_{2}}=\frac{e^{\frac{\mu_{2}-\mu_{1}}{\lambda_{2}}}\left(\lambda_{1}\left(\lambda_{2}+\mu_{1}-\mu_{2}\right)-\lambda_{2}\left(\lambda_{2}-\mu_{1}+\mu_{2}\right)\right)}{\lambda_{2}\left(\lambda_{1}+\lambda_{2}\right)^{3}} I\left[\mu_{1}>\mu_{2}\right] \\
& +\frac{e^{\frac{\mu_{1}-\mu_{2}}{\lambda_{1}}}\left(-\lambda_{1}\left(\lambda_{2}-\mu_{1}+\mu_{2}\right)+\lambda_{2}\left(\mu_{1}-\mu_{2}\right)+\lambda_{1}^{2}\right)}{\lambda_{1}\left(\lambda_{1}+\lambda_{2}\right)^{3}} I\left[\mu_{1}<\mu_{2}\right] \\
& R_{14}=R_{41}=\frac{\partial^{2} \hat{R}^{*}}{\partial \lambda_{1} \partial \mu_{2}}=\frac{e^{\frac{\mu_{2}-\mu_{1}}{\lambda_{2}}}}{\left(\lambda_{1}+\lambda_{2}\right)^{2}} I\left[\mu_{1}>\mu_{2}\right]+\frac{e^{\frac{\mu_{1}-\mu_{2}}{\lambda_{1}}}\left(\lambda_{1} \mu_{1}+\lambda_{2} \mu_{1}-\left(\lambda_{1}+\lambda_{2}\right) \mu_{2}+\lambda_{1}^{2}\right)}{\lambda_{1}^{2}\left(\lambda_{1}+\lambda_{2}\right)^{2}} I\left[\mu_{1}<\mu_{2}\right], \\
& R_{22}=\frac{\partial^{2} \hat{R}^{*}}{\partial \mu_{1}^{2}}=-\frac{e^{\frac{\mu_{2}-\mu_{1}}{\lambda_{2}}}}{\lambda_{2}\left(\lambda_{1}+\lambda_{2}\right)} I\left[\mu_{1}>\mu_{2}\right]-\frac{e^{\frac{\mu_{1}-\mu_{2}}{\lambda_{1}}}}{\lambda_{1}\left(\lambda_{1}+\lambda_{2}\right)} I\left[\mu_{1}<\mu_{2}\right], \\
& R_{23}=R_{32}=\frac{\partial^{2} \hat{R}^{*}}{\partial \lambda_{2} \partial \mu_{1}}=\frac{e^{\frac{\mu_{2}-\mu_{1}}{\lambda_{2}}}\left(\lambda_{1}\left(\mu_{1}-\mu_{2}\right)-\lambda_{2}\left(\lambda_{2}-\mu_{1}+\mu_{2}\right)\right)}{\lambda_{2}^{2}\left(\lambda_{1}+\lambda_{2}\right)^{2}} I\left[\mu_{1}>\mu_{2}\right]-\frac{e^{\frac{\mu_{1}-\mu_{2}}{\lambda_{1}}}}{\left(\lambda_{1}+\lambda_{2}\right)^{2}} I\left[\mu_{1}<\mu_{2}\right], \\
& R_{24}=R_{42}=\frac{\partial^{2} \hat{R}^{*}}{\partial \mu_{2} \partial \mu_{1}}=\frac{e^{\frac{\mu_{2}-\mu_{1}}{\lambda_{2}}}}{\lambda_{2}\left(\lambda_{1}+\lambda_{2}\right)} I\left[\mu_{1}>\mu_{2}\right]-\frac{e^{\frac{\mu_{1}-\mu_{2}}{\lambda_{1}}}}{\lambda_{1}\left(\lambda_{1}+\lambda_{2}\right)} I\left[\mu_{1}<\mu_{2}\right] \\
& R_{33}=\frac{\partial^{2} \hat{R}^{*}}{\partial \lambda_{2}^{2}}=\frac{e^{\frac{\mu_{2}-\mu_{1}}{\lambda_{2}}}\left(\lambda_{2}^{2}\left(\mu_{1}-\mu_{2}\right)\left(2 \lambda_{2}-\mu_{1}+\mu_{2}\right)+2 \lambda_{1} \lambda_{2}\left(\lambda_{2}\left(\mu_{1}-\mu_{2}\right)+\lambda_{2}^{2}-\left(\mu_{1}-\mu_{2}\right)^{2}\right)-\lambda_{1}^{2}\left(\mu_{1}-\mu_{2}\right)^{2}\right)}{\lambda_{2}^{3}\left(\lambda_{1}+\lambda_{2}\right)^{3}} I\left[\mu_{1}>\mu_{2}\right] \\
& +\frac{2 \lambda_{1} e^{\frac{\mu_{1}-\mu_{2}}{\lambda_{1}}}}{\left(\lambda_{1}+\lambda_{2}\right)^{3}} I\left[\mu_{1}<\mu_{2}\right], \\
& R_{34}=R_{43}=\frac{\partial^{2} \hat{R}^{*}}{\partial \lambda_{2} \partial \mu_{2}} \\
& =\frac{e^{\frac{\mu_{2}-\mu_{1}}{\lambda_{2}}}\left(\lambda_{1}\left(\mu_{2}-\mu_{1}\right)+\lambda_{2}\left(\lambda_{2}-\mu_{1}+\mu_{2}\right)\right)}{\lambda_{2}^{2}\left(\lambda_{1}+\lambda_{2}\right)^{2}} I\left[\mu_{1}>\mu_{2}\right]+\frac{e^{\frac{\mu_{1}-\mu_{2}}{\lambda_{1}}}\left(\lambda_{1} \mu_{1}+\lambda_{2} \mu_{1}-\left(\lambda_{1}+\lambda_{2}\right) \mu_{2}+\lambda_{1}^{2}\right)}{\lambda_{1}^{2}\left(\lambda_{1}+\lambda_{2}\right)^{2}} I\left[\mu_{1}<\mu_{2}\right], \\
& R_{44}=\frac{\partial^{2} \hat{R}^{*}}{\partial \mu_{2}^{2}}=-\frac{e^{\frac{\mu_{2}-\mu_{1}}{\lambda_{2}}}}{\lambda_{2}\left(\lambda_{1}+\lambda_{2}\right)} I\left[\mu_{1}>\mu_{2}\right]+\frac{e^{\frac{\mu_{1}-\mu_{2}}{\lambda_{1}}}}{\lambda_{1}\left(\lambda_{1}+\lambda_{2}\right)} I\left[\mu_{1}<\mu_{2}\right], \\
& \sigma_{i j}=I_{i j}^{-1} \text {, } \\
& \rho_{1}=\frac{\partial \rho}{\partial \lambda_{1}}=\frac{\lambda_{1}+2}{\lambda_{1}^{2}+4 \lambda_{1}+2}-\frac{1}{\lambda_{1}}-\frac{1}{1+\lambda_{1}}, \\
& \rho_{2}=\frac{\partial \rho}{\partial \mu_{1}}=\frac{\mu_{1}+2}{\mu_{1}^{2}+4 \mu_{1}+2}-\frac{1}{\mu_{1}}-\frac{1}{1+\mu_{1}}, \\
& \rho_{3}=\frac{\partial \rho}{\partial \lambda_{2}}=\frac{\lambda_{2}+2}{\lambda_{2}^{2}+4 \lambda_{2}+2}-\frac{1}{\lambda_{2}}-\frac{1}{1+\lambda_{2}},
\end{aligned}
$$




$$
\begin{aligned}
& \rho_{4}=\frac{\partial \rho}{\partial \mu_{2}}=\frac{\mu_{2}+2}{\mu_{2}^{2}+4 \mu_{2}+2}-\frac{1}{\mu_{2}}-\frac{1}{1+\mu_{2}}, \\
& R_{1}= \frac{\partial R}{\partial \lambda_{1}}=-\frac{\lambda_{1} e^{\frac{\mu_{1}-\mu_{2}}{\lambda_{1}}}}{\left(\lambda_{1}+\lambda_{2}\right)^{2}}+\frac{e^{\frac{\mu_{1}-\mu_{2}}{\lambda_{1}}}}{\lambda_{1}+\lambda_{2}}-\frac{\left(\mu_{1}-\mu_{2}\right) e^{\frac{\mu_{1}-\mu_{2}}{\lambda_{1}}}}{\lambda_{1}\left(\lambda_{1}+\lambda_{2}\right)} I\left(\mu_{1}<\mu_{2}\right)+\frac{\lambda_{2} e^{\frac{\mu_{2}-\mu_{1}}{\lambda_{2}}}}{\left(\lambda_{1}+\lambda_{2}\right)^{2}} I\left(\mu_{1}>\mu_{2}\right), \\
& R_{2}=\frac{\partial R}{\partial \mu_{1}}=\frac{e^{\frac{\mu_{1}-\mu_{2}}{\lambda_{1}}}}{\lambda_{1}+\lambda_{2}} I\left(\mu_{1}<\mu_{2}\right)+\frac{e^{\frac{\mu_{2}-\mu_{1}}{\lambda_{2}}}}{\lambda_{1}+\lambda_{2}} I\left(\mu_{1}>\mu_{2}\right), \\
& R_{3}=\frac{\partial R}{\partial \lambda_{2}}=-\frac{\lambda_{1} e^{\frac{\mu_{1}-\mu_{2}}{\lambda_{1}}}}{\left(\lambda_{1}+\lambda_{2}\right)^{2}} I\left(\mu_{1}<\mu_{2}\right)+\frac{\lambda_{2} e^{\frac{\mu_{2}-\mu_{1}}{\lambda_{2}}}}{\left(\lambda_{1}+\lambda_{2}\right)^{2}}+\frac{\left(\mu_{2}-\mu_{1}\right) e^{\frac{\mu_{2}-\mu_{1}}{\lambda_{2}}}}{\lambda_{2}\left(\lambda_{1}+\lambda_{2}\right)}-\frac{e^{\frac{\mu_{2}-\mu_{1}}{\lambda_{2}}}}{\lambda_{1}+\lambda_{2}} I\left(\mu_{1}>\mu_{2}\right), \\
& R_{4}=\frac{\partial R}{\partial \mu_{2}}=-\frac{e^{\frac{\mu_{1}-\mu_{2}}{\lambda_{1}}}}{\lambda_{1}+\lambda_{2}} I\left(\mu_{1}<\mu_{2}\right)-\frac{e^{\frac{\mu_{2}-\mu_{1}}{\lambda_{2}}}}{\lambda_{1}+\lambda_{2}} I\left(\mu_{1}>\mu_{2}\right), \\
& L_{111}=\frac{\partial^{3} l^{*}(\Theta)}{\partial \lambda_{1}^{3}}=\frac{2(n-1)}{\lambda_{1}^{3}}, \\
& L_{333}=\frac{\partial^{3} l^{*}(\Theta)}{\partial \lambda_{2}^{3}}=\frac{2(m-1)}{\lambda_{2}^{3}}, \\
& L_{121}=L_{211}=L_{112}=\frac{\partial^{3} l^{*}(\Theta)}{\partial \lambda_{1}^{2} \partial \mu_{1}}=\frac{2 n}{\lambda_{1}^{3}}, \\
& L_{433}=L_{334}=L_{343}=\frac{\partial^{3} l^{*}(\Theta)}{\partial \lambda_{2}^{2} \partial \mu_{2}}=\frac{2 m}{\lambda_{2}^{3}}, \\
& L_{221}=L_{122}=L_{212}=\frac{\partial^{3} l^{*}(\Theta)}{\partial \lambda_{1} \partial \mu_{1}^{2}}=\frac{2 n(n-1)}{\lambda_{1}^{3}}, \\
& L_{443}=L_{344}=L_{434}=\frac{\partial^{3} l^{*}(\Theta)}{\partial \lambda_{2} \partial \mu_{2}^{2}}=\frac{2 m(m-1)}{\lambda_{2}^{3}} .
\end{aligned}
$$

Note: other $L_{i j k}=0$.

\section{Different estimators of $\boldsymbol{R}$ for incomplete data}

It is very difficult to get a complete data and the data are often incomplete. In medical, industrial or others fields researchers might stop their experiment before all items fail because they often do not have time to observe the lifetime of all items in the study or items fail due to random causes. Consequently, researchers use censored data. There are several types of censored data (Type I and II) including right, left, interval, and truncation censoring. Saracoglu et al. (2012) combine type II censoring and a progressive censoring scheme called progressive type II censoring. This scheme allows the researcher to remove active units during the experiment and is defined as: Given $m<n$, and $P_{1}, \ldots, P_{m}$ non-negative integers such that $P_{1}+\cdots+P_{m}=n-m$. Where $n$ stands for the items are on the life test at the same time. At the time of the first failure, one chooses randomly $P_{1}$ items from the rest of the active $n-1$ and then discards. In a similar way, at time of the second failure, one selects $P_{2}$ out of $n-P_{1}-2$ remaining items at random and consequently remove it. Eventually, at the time of the $m^{\text {th }}$ failure, all the remaining active items are removed (see Saracoglu et al. (2012) and references for the advantages of this censoring scheme). 


\subsection{Maximum penalized likelihood estimator of $R$ for incomplete data}

In this subsection, maximum penalized likelihood estimator of $R$ is obtained for progressive type II censored data as follows:

\section{Algorithm 3.}

1. Suppose two progressive censoring schemes $\left(n_{1}, m_{1}, P_{1}, \ldots, P_{m_{1}}\right)$ and $\left(n_{2}, m_{2}, Q_{1}, \ldots, Q_{m_{2}}\right)$ for two independent random variables $X$ and $Y$ with two parameter negative exponential distribution $\operatorname{NED}\left(\lambda_{1}, \mu_{1}\right)$ and $\operatorname{NED}\left(\lambda_{2}, \mu_{2}\right)$ respectively.

2. Suppose two progressively censored samples as $X=\left(X_{1: m_{1}: n_{1}}, \ldots, X_{m_{1}, m_{1}, n_{1}}\right)$ from strength variable $X$ and $Y=\left(Y_{1: m_{2}: n_{2}}, \ldots, Y_{m_{2}, m_{2}, n_{2}}\right)$ from stress variable $Y$.

3. Find the joint density probability functions of $X=\left(X_{1: m_{1}: n_{1}}, \ldots, X_{m_{1}, m_{1}, n_{1}}\right)$ and $Y=\left(Y_{1: m_{2}: n_{2}}, \ldots\right.$, $\left.Y_{m_{2}, m_{2}, n_{2}}\right)$ as:

$$
\begin{aligned}
f_{\left(X_{1: m_{1}: n_{1}}, \ldots, X_{m_{1}, m_{1}, n_{1}}\right)}\left(x_{1}, \ldots, x_{m_{1}}\right) & =c_{1} \prod_{i=1}^{m_{1}} f\left(x_{1}\right)\left(1-F\left(x_{i}\right)^{P_{i}},\right. \\
f_{\left(Y_{1: m_{2}: n_{2}}, \ldots, Y_{m_{2}, m_{2}, n_{2}}\right)}\left(y_{1}, \ldots, y_{m_{2}}\right) & =c_{2} \prod_{j=1}^{m_{2}} f\left(y_{1}\right)\left(1-F\left(y_{j}\right)^{Q_{j}},\right.
\end{aligned}
$$

where

$$
\begin{aligned}
& c_{1}=n_{1}\left(n_{1}-P_{1}-1\right) \cdots\left(n_{1}-m_{1}-P_{1}-\cdots-P_{m_{1}-1}+1\right), \\
& c_{2}=n_{2}\left(n_{2}-Q_{2}-1\right) \cdots\left(n_{2}-m_{2}-Q_{2}-\cdots-Q_{m_{2}-1}+1\right)
\end{aligned}
$$

are normalizing constants see Balakrishnan and Aggarwala (2000).

4. Find the penalized likelihood function

$$
L^{p r}(\Theta)=c_{1} c_{2}\left(x_{1: m_{1}}-\mu_{1}\right)\left(y_{1: m_{2}}-\mu_{2}\right) \prod_{i=1}^{m_{1}} f\left(x_{1}\right)\left(1-F\left(x_{i}\right)^{P_{i}} \prod_{j=1}^{m_{2}} f\left(y_{1}\right)\left(1-F\left(y_{j}\right)^{Q_{j}} .\right.\right.
$$

5. Taking the logarithm of penalized likelihood function

$$
\begin{aligned}
l^{p r}(\Theta)= & \log c_{1}+\log c_{2}+\log \left(x_{1: m_{1}-\mu_{1}}\right)+\log \left(y_{1: m_{2}-\mu_{2}}\right)-m_{1} \log \lambda_{1}-m_{2} \log \lambda_{2} \\
& -\frac{1}{\lambda_{1}} \sum_{i=1}^{m_{1}}\left(P_{i}+1\right)\left(x_{i}-\mu_{1}\right)-\frac{1}{\lambda_{2}} \sum_{j=1}^{m_{2}}\left(Q_{j}+1\right)\left(y_{j}-\mu_{2}\right) .
\end{aligned}
$$

6. Find maximum penalized likelihood estimators of $\Theta=\left(\lambda_{1}, \lambda_{2}, \mu_{1}, \mu_{2}\right)$ as

$$
\begin{aligned}
& \lambda_{1}^{p r}=\frac{1}{m_{1}}\left[\sum_{i=1}^{m_{1}}\left(P_{i}+1\right) x_{i}-x_{1: m_{1}} \sum_{i=1}^{m_{1}}\left(P_{i}+1\right)\right], \\
& \lambda_{2}^{p r}=\frac{1}{m_{2}}\left[\sum_{j=1}^{m_{2}}\left(Q_{j}+1\right) y_{j}-y_{2: m_{2}} \sum_{j=1}^{m_{2}}\left(Q_{j}+1\right)\right],
\end{aligned}
$$




$$
\begin{aligned}
& \mu_{1}^{p r}=x_{1: m_{1}}-\frac{\sum_{i=1}^{m_{1}}\left(P_{i}+1\right) x_{i}-x_{1: m_{1}} \sum_{i=1}^{m_{1}}\left(P_{i}+1\right)}{\left(m_{1}-1\right) \sum_{i=1}^{m_{1}}\left(P_{i}+1\right)}, \\
& \mu_{2}^{p r}=y_{1: m_{2}}-\frac{\sum_{j=1}^{m_{2}}\left(Q_{j}+1\right) y_{j}-y_{1: m_{2}} \sum_{j=1}^{m_{2}}\left(Q_{j}+1\right)}{\left(m_{2}-1\right) \sum_{j=1}^{m_{2}}\left(Q_{j}+1\right)} .
\end{aligned}
$$

7. Find maximum penalized likelihood estimator of $R$ says $R^{p r}$ using the invariance property as:

- Case 1: $\mu_{1}=\mu_{2}$, then

$$
R^{p r}=\frac{\lambda_{1}^{p r}}{\lambda_{1}^{p r}+\lambda_{2}^{p r}}
$$

- Case 2: $\mu_{1} \neq \mu_{2}$, then

$$
R^{p r}=\left[1-\frac{\lambda_{2}^{p r}}{\lambda_{1}^{p r}+\lambda_{2}^{p r}} e^{\frac{\mu_{2}^{p r}-\mu_{1}^{p r}}{\lambda_{2}^{p r}}}\right] I\left(\mu_{1}^{p r}>\mu_{2}^{p r}\right)+\frac{\lambda_{1}^{p r}}{\lambda_{1}^{p r}+\lambda_{2}^{p r}} e^{\frac{\left(\mu_{1}^{p r}-\mu_{2}^{p r}\right)}{p_{2}^{p r}}} I\left(\mu_{1}^{p r}<\mu_{2}^{p r}\right) .
$$

Now to find the asymptotic $100(1-\alpha) \%$ confidence interval for $R$ is given by

$$
R \in\left(R^{p r} \pm \frac{Z_{\frac{\alpha}{2}}}{\sqrt{n+m}} \sqrt{\widehat{\operatorname{Var}}\left(R^{p r}\right)}\right)
$$

where $Z_{\alpha / 2}$ is the upper $(\alpha / 2)^{\text {th }}$ quantile of standard normal distribution and $\widehat{\operatorname{Var}}\left(R^{p r}\right)$ is the value of $\operatorname{Var}\left(R^{p r}\right)$ at the penalized maximum likelihood estimators of parameters $\Theta=\left(\lambda_{1}, \mu_{1}, \lambda_{2}, \mu_{2}\right)$, where

$$
\operatorname{Var}\left(R^{p r}\right)=\sum_{j=1}^{4} \sum_{i=1}^{4} \frac{\partial R}{\partial \Theta_{i}} \frac{\partial R}{\partial \Theta_{j}}\left(I_{i j}^{-1}\right)^{p r},
$$

where $\Theta=\left(\lambda_{1}, \mu_{1}, \lambda_{2}, \mu_{2}\right)$ and $I_{i j}^{-1}$ is the $(i, j)^{t h}$ element of the inverse of the Fisher information matrix $I_{i j}$ given by

$$
\begin{aligned}
& I_{i j}^{p r}=\left(\begin{array}{llll}
E\left[\frac{-\partial^{2} p^{r r}(\Theta)}{\partial \lambda_{1}^{2}}\right] & E\left[\frac{-\partial^{2} p^{r r}(\Theta)}{\partial \lambda_{1} \partial \partial_{1}}\right] & E\left[\frac{-\partial^{2} p^{r r}(\Theta)}{\partial \lambda_{1} \partial \partial_{2}}\right] & E\left[\frac{-\partial^{2} p^{r r}(\Theta)}{\partial \lambda_{1} \partial \mu_{2}}\right] \\
E\left[\frac{-\partial^{2} p^{r}(\Theta)}{\partial \mu_{1} \partial \lambda_{1}}\right] & E\left[\frac{-\partial^{2} p^{r}(\Theta)}{\partial \mu_{1}^{2}}\right] & E\left[\frac{-\partial^{2} p^{r}(\Theta)}{\partial \mu_{1} \partial \lambda_{2}}\right] & E\left[\frac{-\partial^{2} p^{r}(\Theta)}{\partial \mu_{1} \partial \mu_{2}}\right] \\
E\left[\frac{-\partial^{2} p^{r}(\Theta)}{\partial \lambda_{2} \partial \lambda_{1}}\right] & E\left[\frac{-\partial^{2} p^{r}(\Theta)}{\partial \lambda_{2} \partial \mu_{1}}\right] & E\left[\frac{-\partial^{2} p^{r}(\Theta)}{\partial \lambda_{2}^{2}}\right] & E\left[\frac{-\partial^{2} p^{r}(\Theta)}{\partial \lambda_{2} \partial \mu_{2}}\right] \\
E\left[\frac{-\partial^{2} p^{r}(\Theta)}{\partial \mu_{2} \partial \lambda_{1}}\right] & E\left[\frac{-\partial^{2} p^{r}(\Theta)}{\partial \mu_{2} \partial \mu_{1}}\right] & E\left[\frac{-\partial^{2} p^{r}(\Theta)}{\partial \mu_{2} \partial \lambda_{2}}\right] & E\left[\frac{-\partial^{2} p^{r}(\Theta)}{\partial \mu_{2}^{2}}\right]
\end{array}\right), \\
& \left(I_{i j}^{-1}\right)^{p r}=\left(\begin{array}{llll}
\operatorname{Var}\left(\lambda_{1}^{p r}\right) & \operatorname{Cov}\left(\lambda_{1}^{p r}, \mu_{1}^{p r}\right) & \operatorname{Cov}\left(\lambda_{1}^{p r}, \lambda_{2}^{p r}\right) & \operatorname{Cov}\left(\lambda_{1}^{p r}, \mu_{2}^{p r}\right) \\
\operatorname{Cov}\left(\mu_{1}^{p r}, \lambda_{1}^{p r}\right) & \operatorname{Var}\left(\mu_{1}^{p r}\right) & \operatorname{Cov}\left(\mu_{1}^{p r}, \lambda_{2}^{p r}\right) & \operatorname{Cov}\left(\mu_{1}^{p r}, \mu_{2}^{p r}\right) \\
\operatorname{Cov}\left(\lambda_{2}^{p r}, \lambda_{1}^{p r}\right) & \operatorname{Cov}\left(\lambda_{2}^{p r}, \mu_{1}^{p r}\right) & \operatorname{Var}\left(\lambda_{2}^{p r}\right) & \operatorname{Cov}\left(\lambda_{2}^{p r}, \mu_{2}^{p r}\right) \\
\operatorname{Cov}\left(\mu_{2}^{p r}, \lambda_{1}^{p r}\right) & \operatorname{Cov}\left(\mu_{2}^{p r}, \mu_{1}^{p r}\right) & \operatorname{Cov}\left(\mu_{2}^{p r}, \lambda_{2}^{p r}\right) & \operatorname{Var}\left(\mu_{2}^{p r}\right)
\end{array}\right),
\end{aligned}
$$


and

$$
\begin{aligned}
& \operatorname{Var}\left(\lambda_{1}^{p r}\right)=\frac{2\left(m_{1}-1\right) \sum_{i=1}^{m_{1}}\left(1+P_{i}\right)-m_{1}^{2}}{\lambda_{1}^{2} m_{1}^{2}}, \quad \operatorname{Var}\left(\lambda_{2}^{p r}\right)=\frac{2\left(m_{2}-1\right) \sum_{j=1}^{m_{2}}\left(1+Q_{j}\right)-m_{2}^{2}}{\lambda_{2}^{2} m_{2}^{2}}, \\
& \operatorname{Var}\left(\mu_{1}^{p r}\right)=\frac{\lambda_{1}^{2}}{n(n-1)}, \quad \operatorname{Var}\left(\mu_{2}^{p r}\right)=\frac{\lambda_{2}^{2}}{m(m-1)}, \\
& \operatorname{Cov}\left(\lambda_{1}^{p r}, \mu_{1}^{p r}\right)=\operatorname{Cov}\left(\mu_{1}^{p r}, \lambda_{1}^{p r}\right)=\frac{\lambda_{1}^{2}}{\sum_{i=1}^{m_{1}}\left(1+P_{i}\right)}, \quad \operatorname{Cov}\left(\lambda_{1}^{p r}, \lambda_{2}^{p r}\right)=\operatorname{Cov}\left(\lambda_{2}^{p r}, \lambda_{1}^{p r}\right)=0, \\
& \operatorname{Cov}\left(\lambda_{1}^{p r}, \mu_{2}^{p r}\right)=\operatorname{Cov}\left(\mu_{2}^{p r}, \lambda_{1}^{p r}\right)=0, \quad \operatorname{Cov}\left(\mu_{1}^{p r}, \lambda_{2}^{p r}\right)=\operatorname{Cov}\left(\lambda_{2}^{p r}, \mu_{1}^{p r}\right)=0, \\
& \operatorname{Cov}\left(\mu_{1}^{p r}, \mu_{2}^{p r}\right)=\operatorname{Cov}\left(\mu_{2}^{p r}, \mu_{1}^{p r}\right)=0, \quad \operatorname{Cov}\left(\lambda_{2}^{p r}, \mu_{2}^{p r}\right)=\operatorname{Cov}\left(\mu_{2}^{p r}, \lambda_{2}^{p r}\right)=\frac{\lambda_{2}^{2}}{\sum_{j=1}^{m_{2}}\left(1+Q_{j}\right)} .
\end{aligned}
$$

\subsection{Bayes estimation and credible interval $R$ for incomplete data}

To find the Bayes estimator of $R$ as:

Assume all parameters $\lambda_{1}, \lambda_{2}, \mu_{1}, \mu_{2}$ are random variables having gamma prior distributions with parameters $\left(a_{i}, b_{i}\right), i=1,2,3,4$ respectively. Where

$$
\begin{array}{ll}
\pi\left(\lambda_{1}\right)=\frac{b_{1}^{a_{1}}}{\Gamma\left(a_{1}\right)} \lambda_{1}^{a_{1}-1} e^{-\lambda_{1} b_{1}}, & \lambda_{1}, a_{1}, b_{1}>0, \\
\pi\left(\lambda_{2}\right)=\frac{b_{2}^{a_{2}}}{\Gamma\left(a_{2}\right)} \lambda_{2}^{a_{2}-1} e^{-\lambda_{2} b_{2}}, & \lambda_{2}, a_{2}, b_{2}>0, \\
\pi\left(\mu_{1}\right)=\frac{b_{3}^{a_{3}}}{\Gamma\left(a_{3}\right)} \mu_{1}^{a_{3}-1} e^{-\mu_{1} b_{3}}, & \mu_{1}, a_{3}, b_{3}>0, \\
\pi\left(\mu_{2}\right)=\frac{b_{4}^{a_{4}}}{\Gamma\left(a_{4}\right)} \mu_{2}^{a_{4}-1} e^{-\mu_{2} b_{4}}, & \mu_{2}, a_{4}, b_{4}>0 .
\end{array}
$$

Then the joint posterior density function of $\lambda_{1}, \lambda_{2}, \mu_{1}, \mu_{2}$ is given by

$$
L\left(\lambda_{1}, \lambda_{2}, \mu_{1}, \mu_{2} \mid \text { data }\right)=\frac{L\left(\text { data } \mid \lambda_{1}, \lambda_{2}, \mu_{1}, \mu_{2}\right) \pi\left(\lambda_{1}\right) \pi\left(\lambda_{2}\right) \pi\left(\mu_{1}\right) \pi\left(\mu_{2}\right)}{\int_{0}^{\infty} \int_{0}^{\infty} \int_{0}^{\infty} \int_{0}^{\infty} L\left(\text { data } \mid \lambda_{1}, \lambda_{2}, \mu_{1}, \mu_{2}\right) \pi\left(\lambda_{1}\right) \pi\left(\lambda_{2}\right) \pi\left(\mu_{1}\right) \pi\left(\mu_{2}\right) d \lambda_{1} d \lambda_{2} d \pi\left(\mu_{1}\right) d \pi\left(\mu_{2}\right)} .
$$

But the joint posterior density function can not compute analytically. So we use MCMC technique to compute Bayes estimator of $R$ and construct the corresponding credible interval.

Now compute the Bayes estimator using MCMC technique when $\mu_{1}=\mu_{2}$ and under the assumption that the parameters $\lambda_{1}, \lambda_{2}, \mu$ have independent inverse gamma (IG) priors with the probability density functions as:

$$
\begin{array}{ll}
\pi_{1}\left(\lambda_{1} \mid a_{1}, b_{1}\right)=\frac{b_{1}^{a_{1}}}{\Gamma\left(a_{1}\right)} \lambda_{1}^{-a_{1}-1} e^{\frac{-b_{1}}{\lambda_{1}}}, & \lambda_{1}>0, \\
\pi_{2}\left(\lambda_{2} \mid a_{2}, b_{2}\right)=\frac{b_{2}^{a_{2}}}{\Gamma\left(a_{2}\right)} \lambda_{2}^{-a_{2}-1} e^{\frac{-b_{2}}{\lambda_{2}}}, & \lambda_{2}>0, \\
\pi_{3}\left(\mu \mid a_{3}, b_{3}\right)=\frac{b_{3}^{a_{3}}}{\Gamma\left(a_{3}\right)} \mu^{-a_{3}-1} e^{\frac{-b_{3}}{\mu}}, & \mu>0,
\end{array}
$$


where $a_{1}, a_{2}, a_{3}, b_{1}, b_{2}, b_{3}$ are chosen to reflect prior knowledge about $\lambda_{1}, \lambda_{2}, \mu$. The posterior probability density functions of $\lambda_{1}, \lambda_{2}$ are

$$
\begin{aligned}
& \lambda_{1} \mid \mu, \lambda_{2}, \text { data } \sim \mathrm{IG}\left(m_{1}+a_{1}, b_{1}+\sum_{i=1}^{m_{1}}\left(P_{i}+1\right)\left(x_{i}-\mu\right)\right), \\
& \lambda_{2} \mid \mu, \lambda_{1}, \text { data } \sim \mathrm{IG}\left(m_{2}+a_{2}, b_{2}+\sum_{j=1}^{m_{2}}\left(Q_{j}+1\right)\left(y_{j}-\mu\right)\right)
\end{aligned}
$$

and

$$
f\left(\mu \mid \lambda_{1}, \lambda_{2}, \text { data }\right) \propto\left(x_{1: m_{1}}-\mu\right)\left(y_{1: m_{2}}-\mu\right)\left(\frac{1}{\mu}\right)^{a_{3}-1} \exp \left[-\frac{b_{3}}{\mu}-\frac{1}{\lambda_{1}} \sum_{i=1}^{m_{1}}\left(P_{i}+1\right)\left(x_{i}-\mu\right)-\frac{1}{\lambda_{2}} \sum_{j=1}^{m_{2}}\left(Q_{j}+1\right)\left(y_{j}-\mu\right)\right] .
$$

The posterior probability density function of $\mu$ is unknown, but we assume it is a normal distribution and use Metropolis-Hastings algorithm to find the Bayes estimator as:

\section{Algorithm 4.}

1. Start with an initial guess $\lambda_{1}^{(0)}, \lambda_{2}^{(0)}, \mu^{(0)}$.

2. Set $t=1$.

3. Using Metropolis-Hastings, generate $\mu^{(t)}$ from $f\left(\mu \mid \lambda_{1}, \lambda_{2}\right.$, data) using Metropolis-Hastings algorithm with the proposal distribution $N\left(\mu^{t-1}, 1\right)$.

4. Generate $\lambda_{1}^{(t)}$ from $\operatorname{IG}\left(m_{1}+a_{1}, b_{1}+\sum_{i=1}^{m_{1}}\left(P_{i}+1\right)\left(x_{i}-\mu^{(t-1)}\right)\right)$.

5. Generate $\lambda_{2}^{(t)}$ from $\operatorname{IG}\left(m_{2}+a_{2}, b_{2}+\sum_{j=1}^{m_{2}}\left(Q_{i}+j\right)\left(y_{j}-\mu^{(t-1)}\right)\right)$.

6. Compute $R^{(t)}$.

7. Set $t=t+1$.

8. Repeat steps 3-7, $T$ times.

The Bayes estimator of $R$ says $R_{p r}^{B}$ compute under square error loss function is given by

$$
R_{p r}^{B}=\frac{1}{T-M} \sum_{t=M+1}^{T-M} R^{t}
$$

where $M$ is burn-in period. Also the highest probability density (HPD) $100(1-\gamma) \%$ credible interval is obtained by the method of Chen and Shao (1999).

\section{Simulation study}

In this section a simulation study is performed to compare the performance of estimates of $R$ obtained by using different methods; all computations are obtained using Mathematica 10. 
Table 1: Results of simulation study for complete data

\begin{tabular}{|c|c|c|c|c|c|c|c|c|}
\hline \multirow[t]{2}{*}{$(n, m)$} & \multirow{2}{*}{$\left(\lambda_{1}^{*}, \lambda_{2}^{*}, \mu_{1}^{*}, \mu_{2}^{*}\right)$} & \multicolumn{3}{|c|}{$\begin{array}{l}\text { Maximum penalized } \\
\text { likelihood estimator }\end{array}$} & \multicolumn{3}{|c|}{ Bayes estimator } & \multirow[t]{2}{*}{ ACI } \\
\hline & & $\overrightarrow{R^{*}}$ & Bias & MSE & $R^{B}$ & Bias & MSE & \\
\hline \multicolumn{9}{|c|}{$\left(\lambda_{1}, \lambda_{2}, \mu_{1}, \mu_{2}\right)=(1,1,1.5,1)$ and $R=0.6967, \mu_{1}>\mu_{2}$} \\
\hline$(30,30)$ & $0.0738,0.0520,2.4090,1.9489)$ & 0.9999 & 0.0050 & 0.0015 & 0.9997 & -0.0001 & 0.0000 & $(0.6200,1.0000)$ \\
\hline$(30,50)$ & $.0597,0.0677,2.4450,1.9320)$ & 0.9997 & 0.0037 & 0.0011 & 0.9920 & -0.0004 & 0.0000 & $(0.6101,0.9999)$ \\
\hline$(30,100)$ & $(0.4850,0.0772,2.4505,1.9222)$ & 0.9993 & 0.0023 & 0.0007 & 0.9989 & 0.0003 & 0.0000 & $(0.6011,0.9995)$ \\
\hline$(50,50)$ & $(0.1026,0.0653,2.4016,1.9354)$ & 0.9996 & 0.0030 & 0.0009 & 0.9991 & -0.0004 & 0.0000 & $(0.6412,1.0000)$ \\
\hline$(50,100)$ & $(0.0567,0.0682,2.4444,1.9356)$ & 0.9996 & 0.0020 & 0.0006 & 0.9993 & -0.0002 & 0.0000 & $(0.6454,1.0000)$ \\
\hline$(100,100)$ & $(0.0689,0.0677,2.4232,1.9323)$ & 0.9996 & 0.0015 & 0.0004 & 0.9993 & -0.0002 & 0.0000 & $(0.6600,1.0000)$ \\
\hline \multicolumn{9}{|c|}{$\left(\lambda_{1}, \lambda_{2}, \mu_{1}, \mu_{2}\right)=(1.5,1,1.5,1.5)$ and $R=0.6, \mu_{1}=\mu_{2}$} \\
\hline$(30,30)$ & $(0.1064,0.0659,2.9110,2.4360)$ & 0.6192 & 0.0003 & 0.0000 & 0.6182 & -0.0006 & 0.0000 & $(0.5999,0.6400)$ \\
\hline$(30,50)$ & $(0.0922,0.0764,2.9090,2.4234)$ & 0.5468 & -0.0006 & 0.0000 & 0.5457 & -0.0001 & 0.0000 & $(0.5124,0.7000)$ \\
\hline$(30,100)$ & $(0.1572,0.0612,2.8360,2.4398)$ & 0.7197 & 0.0009 & 0.0001 & 0.7195 & -0.0001 & 0.0000 & $(0.5300,0.7500)$ \\
\hline$(50,50)$ & $(0.1417,0.0751,2.8666,2.4295)$ & 0.6536 & 0.0005 & 0.0000 & 0.6524 & -0.0011 & 0.0000 & $(0.5504,0.7700)$ \\
\hline$(50,100)$ & $(0.1039,0.0565,2.8953,2.4490)$ & 0.6474 & 0.0003 & 0.0000 & 0.6473 & 0.0003 & 0.0000 & $(0.5965,0.7584)$ \\
\hline$(100,100)$ & $(0.0885,0.1064,2.9112,2.3955)$ & 0.4539 & -0.0007 & 0.0001 & 0.4519 & -0.0007 & 0.0000 & $(0.4210,0.7145)$ \\
\hline \multicolumn{9}{|c|}{$\left(\lambda_{1}, \lambda_{2}, \mu_{1}, \mu_{2}\right)=(1,1.5,1.5,2)$ and $R=0.2420, \mu_{1}<\mu_{2}$} \\
\hline$(100,100)$ & $(0.0838,0.1059,2.4260,3.3815)$ & 0.0001 & 0.2419 & 0.4419 & 0.0016 & 0.1622 & 0.4386 & $(0.0001,0.2500)$ \\
\hline
\end{tabular}

\subsection{Simulation study for complete data}

1. For given values of $\left(\lambda_{1}, \lambda_{2}, \mu_{1}, \mu_{2}\right)$ compute the true value of $R$ from (2.1) or (2.2).

2. For given $(n, m)$ generate sample from size $n$ from (1.1) with given parameters $\left(\lambda_{1}, \mu_{1}\right)$ and generate sample from size $m$ from (1.1) with given parameters $\left(\lambda_{2}, \mu_{2}\right)$.

3. Evaluate maximum penalized likelihood estimators of $\left(\lambda_{1}, \lambda_{2}, \mu_{1}, \mu_{2}\right)$ and $R$ using Algorithm 1.

4. Repeat step 2-3 $N=10^{4}$ times and calculate the biases and MSEs.

5. Calculate the asymptotic confidence interval (ACI) of $R$ according to (3.1).

6. Using 2-4 to compute $R^{B}$ using Algorithm 2.

7. Calculate the biases and MSEs for the Bayes estimator.

Table 1 shows the simulation study results for the complete data. We observe that for large samples size the estimator values of $\hat{R}^{*}$ and $R^{B}$ became constant, when $\mu_{1}=\mu_{2}$ the estimator values of $R$ nearest of its true vales. $R$ has bad estimator when $\mu_{1}<\mu_{2}$, also biases and MSE's decrease when sample sizes increase.

\subsection{Simulation study for incomplete data}

In this subsection we compare the maximum penalized likelihood estimator and Bayes estimator using the MCMC technique of $R$ in bias, MSE as well as given the ACI and HPD credible intervals for progressive type II censored data as:

1. For different parameter values $\left(\lambda_{1}, \lambda_{2}, \mu_{1}, \mu_{2}\right)=(1,1,1.5,0.5),(1,1,1.5,1.5)$, and $(1,1,2.5,2.5)$. 
Table 2: Results of simulation study for incomplete data (biases and MSE's) of maximum penalized likelihood estimator and Bayes estimator of $R$

\begin{tabular}{|c|c|c|c|c|c|c|c|c|c|c|c|c|}
\hline \multirow{3}{*}{$\begin{array}{c}\mathrm{CS} \\
(P, Q)\end{array}$} & \multirow{2}{*}{\multicolumn{3}{|c|}{$\begin{array}{l}\text { Maximum penalized } \\
\text { likelihood estimator }\end{array}$}} & \multicolumn{9}{|c|}{ Bayes estimator } \\
\hline & & & & \multicolumn{3}{|c|}{ Prior 1} & \multicolumn{3}{|c|}{ Prior 2} & \multicolumn{3}{|c|}{ Prior 3} \\
\hline & $R^{p r}$ & Bias & MSE & $R_{p r}^{B}$ & Bias & MSE & $R_{p r}^{B}$ & Bias & MSE & $R_{p r}^{B}$ & Bias & MSE \\
\hline \multicolumn{13}{|c|}{$\left(\lambda_{1}, \lambda_{2}, \mu_{1}, \mu_{2}\right)=(1,1,0.5,0.5)$} \\
\hline$(\mathrm{I}, \mathrm{I})$ & 0.5520 & 0.0026 & 0.0001 & 0.5001 & -0.0001 & 0.0000 & 0.5108 & -0.0108 & 0.0001 & 0.5027 & -0.0027 & 0.0000 \\
\hline (I, II) & 0.3480 & -0.0075 & 0.0011 & 0.4874 & 0.0126 & 0.0001 & 0.4840 & 0.0160 & 0.0002 & 0.5149 & -0.0149 & 0.0002 \\
\hline (I, III) & 0.5338 & 0.0016 & 0.0000 & 0.4951 & 0.0049 & 0.0000 & 0.5058 & -0.0058 & 0.0000 & 0.4988 & 0.0012 & 0.0000 \\
\hline (II, II) & 0.3125 & -0.0093 & 0.0017 & 0.4977 & 0.0023 & 0.0000 & 0.4922 & 0.0078 & 0.0000 & 0.4872 & 0.0128 & 0.0001 \\
\hline (II, III) & 0.5245 & 0.0012 & 0.0000 & 0.4942 & 0.0058 & 0.0000 & 0.5135 & -0.0135 & 0.0001 & 0.4816 & 0.0184 & 0.0003 \\
\hline (III, III) & 0.4017 & -0.0049 & 0.0004 & 0.5132 & -0.0132 & 0.0001 & 0.4822 & 0.0178 & 0.0003 & 0.4992 & 0.0008 & 0.0000 \\
\hline \multicolumn{13}{|c|}{$\left(\lambda_{1}, \lambda_{2}, \mu_{1}, \mu_{2}\right)=(1,1,1.5,1.5)$} \\
\hline$(\mathrm{I}, \mathrm{I})$ & 0.5753 & 0.0037 & 0.0002 & 0.4790 & 0.0209 & 0.0004 & 0.5064 & -0.0064 & 0.0000 & 0.5023 & -0.0023 & 0.0000 \\
\hline (I, II) & 0.7423 & 0.0121 & 0.0029 & 0.4919 & 0.0082 & 0.0000 & 0.4788 & 0.0211 & 0.0004 & 0.4917 & 0.0082 & 0.0006 \\
\hline (I, III) & 0.5731 & 0.0036 & 0.0029 & 0.5065 & -0.0065 & 0.0000 & 0.5280 & -0.0280 & 0.0007 & 0.4847 & 0.0152 & 0.0002 \\
\hline (II, II) & 0.3524 & -0.0073 & 0.0010 & 0.5108 & -0.0106 & 0.0001 & 0.5013 & -0.0013 & 0.0000 & 0.4968 & 0.0031 & 0.0000 \\
\hline (II, III) & 0.3085 & -0.0057 & 0.0006 & 0.4850 & 0.0144 & 0.0003 & 0.4904 & 0.0095 & 0.0000 & 0.5106 & 0.0106 & 0.0001 \\
\hline (III, III) & 0.3461 & -0.0076 & 0.0011 & 0.5185 & -0.0185 & 0.0003 & 0.5220 & -0.0220 & 0.0004 & 0.5152 & -0.0192 & 0.0002 \\
\hline \multicolumn{13}{|c|}{$\left(\lambda_{1}, \lambda_{2}, \mu_{1}, \mu_{2}\right)=(1,1,2.5,2.5)$} \\
\hline$(\mathrm{I}, \mathrm{I})$ & 0.6344 & 0.0067 & 0.0009 & 0.4820 & 0.0179 & 0.0003 & 0.4780 & 0.0219 & 0.0004 & 0.4958 & 0.0041 & 0.0000 \\
\hline (I, II) & 0.5930 & 0.0046 & 0.0004 & 0.5116 & -0.0116 & 0.0001 & 0.4886 & 0.0113 & 0.0001 & 0.5162 & -0.0162 & 0.0002 \\
\hline (I, III) & 0.5433 & 0.0021 & 0.0000 & 0.4999 & 0.0001 & 0.0000 & 0.5046 & -0.0046 & 0.0000 & 0.4990 & 0.0609 & 0.0000 \\
\hline (II, II) & 0.3009 & -0.0099 & 0.0019 & 0.5094 & -0.0094 & 0.0000 & 0.5023 & -0.0023 & 0.0000 & 0.4933 & 0.0066 & 0.0000 \\
\hline (II, III) & 0.4359 & -0.0032 & 0.0002 & 0.4696 & 0.0303 & 0.0009 & 0.5295 & -0.0295 & 0.0008 & 0.4812 & 0.0187 & 0.0003 \\
\hline (III, III) & 0.5191 & 0.0009 & 0.0000 & 0.5089 & -0.0089 & 0.0000 & 0.5016 & -0.0016 & 0.0000 & 0.4939 & 0.0060 & 0.0000 \\
\hline
\end{tabular}

Also use censoring schemes (CS) as

$$
\begin{aligned}
\mathrm{I} & =(10,30)=(0,0,0,0,0,0,0,0,0,20), \\
\mathrm{II} & =(10,30)=(20,0,0,0,0,0,0,0,0,0), \\
\mathrm{III} & =(10,30)=(2,2,2,2,2,2,2,2,2,2) .
\end{aligned}
$$

2. Find $R^{p r}$ using Algorithm 3.

3. Compute bias and MSE.

4. Compute ACI of (4.1).

5. Find the $R_{p r}^{B}$ and compute HPD credible interval using Algorithm 4 and use censoring schemes in 1 and the following priors

Prior 1. $a_{i}=0, b_{i}=0, \quad i=1,2,3$.

Prior 2. $a_{i}=1, b_{i}=2, \quad i=1,2,3$.

Prior 3. $a_{i}=2, b_{i}=3, \quad i=1,2,3$.

Tables 2 and 3 show results of simulation study of $R$ for incomplete data.

\section{Data analysis}

In this section, we present a real example to sure our proposed model can apply in practice. Badar and Priest (1982) reported the data represent the strength measured in GPA for single carbon fibers, and impregnated 1000-carbon fiber tows. Single fibers were tested under tension at gauge lengths of 20 
Table 3: Comparison between ACI and HPD credible interval of $R$

\begin{tabular}{ccccc}
\hline \hline \multirow{2}{*}{ CS } & \multirow{2}{*}{ ACI } & \multicolumn{3}{c}{ HPD credible interval of $R$} \\
\cline { 3 - 5 } & & $\left(\lambda_{1}, \lambda_{2}, \mu_{1}, \mu_{2}\right)=(1,1,0.5,0.5)$ & Prior 2 \\
\hline (I, I) & $(0.4832,0.6207)$ & $(0.4510,0.5491)$ & $(0.4607,0.5608)$ & $(0.4534,0.5519)$ \\
(I, II) & $(0.3978,0.6021)$ & $(0.4397,0.5352)$ & $(0.4366,0.5314)$ & $(0.4644,0.5638)$ \\
(I, III) & $(0.3490,0.6509)$ & $(0.4466,0.5436)$ & $(0.4562,0.5554)$ & $(0.4499,0.5476)$ \\
(II, II) & $(0.3002,0.6997)$ & $(0.4490,0.5465)$ & $(0.4440,0.5405)$ & $(0.4395,0.5350)$ \\
(II, III) & $(0.4436,0.5563)$ & $(0.4458,0.5426)$ & $(0.4631,0.5638)$ & $(0.4344,0.5288)$ \\
(III, III) & $(0.3803,0.6196)$ & $(0.4629,0.5635)$ & $(0.4349,0.5294)$ & $(0.4503,0.5481)$ \\
\hline & & $\left(\lambda_{1}, \lambda_{2}, \mu_{1}, \mu_{2}\right)=(1,1,1.5,1.5)$ & $(0.4531,0.5515)$ \\
(I, I) & $(0.3894,0.6105)$ & $(0.4320,0.5259)$ & $(0.4568,0.5561)$ & $(0.4436,0.5399)$ \\
(I, II) & $(0.3839,0.6160)$ & $(0.4435,0.5398)$ & $(0.4319,0.5278)$ & $(0.4372,0.5322)$ \\
(I, III) & $(0.3899,0.6105)$ & $(0.4568,0.5561)$ & $(0.4762,0.5797)$ & $(0.4481,0.5455)$ \\
(II, II) & $(0.3685,0.6314)$ & $(0.4608,0.5609)$ & $(0.4522,0.5504)$ & $(0.4606,0.5607)$ \\
(II, III) & $(0.3606,0.6393)$ & $(0.4850,0.5326)$ & $(0.4424,0.5385)$ & $(0.4647,0.5657)$ \\
(III, III) & $(0.3997,0.6002)$ & $(0.4677,0.5193)$ & $(0.4709,0.5732)$ & $(0.4472,0.5443)$ \\
\hline & & $\left(\lambda_{1}, \lambda_{2}, \mu_{1}, \mu_{2}\right)=(1,1,2.5,2.5)$ & $(0.4656,0.5666)$ \\
(I, I) & $(0.4190,0.5809)$ & $(0.4348,0.5293)$ & $(0.4311,0.5248)$ & $(0.4501,0.5479)$ \\
(I, II) & $(0.2338,0.7661)$ & $(0.4615,0.5618)$ & $(0.4407,0.5365)$ & $(0.4449,0.5416)$ \\
(I, III) & $(0.4190,0.5809)$ & $(0.4509,0.5489)$ & $(0.4551,0.5541)$ & $(0.4340,0.5283)$ \\
(II, II) & $(0.3724,0.6275)$ & $(0.4595,0.5593)$ & $(0.4531,0.5515)$ & $(0.4455,0.5423)$ \\
(II, III) & $(0.4389,0.5610)$ & $(0.4236,0.5156)$ & $(0.4776,0.5814)$ & $(0.4524,0.5507)$ \\
(III, III) & $(0.4501,0.5498)$ & $(0.4590,0.5588)$ &
\end{tabular}

$\mathrm{CS}$ = censoring schemes; $\mathrm{ACI}=$ asymptotic confidence interval; HPD = highest probability density.

$\mathrm{mm}$ (data set 1) and $10 \mathrm{~mm}$ (data set 2). These data have been used previously by Raqab and Kundu (2005), Kundu and Gupta (2006), Kundu and Raqab (2009), and Asgharzadeh et al. (2011).

- Data set I: (gauge lengths of $20 \mathrm{~mm}$ ).

1.3121 .3141 .4791 .5521 .7001 .8031 .8611 .8651 .9441 .9581 .9661 .9972 .0062 .0212 .0272 .055 2.0632 .0982 .1402 .1792 .2242 .2402 .2532 .2702 .2722 .2742 .3012 .3012 .3592 .3822 .3822 .426 2.4342 .4352 .4782 .4902 .5112 .5142 .5352 .5542 .5662 .5702 .5862 .6292 .6332 .6422 .6482 .684 2.6972 .7262 .7702 .7732 .8002 .8092 .8182 .8212 .8482 .8802 .9543 .0123 .0673 .0843 .0903 .096 3.1283 .2333 .4333 .5853 .585

- Data set II (gauge lengths of $10 \mathrm{~mm}$ ).

1.9012 .1322 .2032 .2282 .2572 .3502 .3612 .3962 .3972 .4452 .4542 .4742 .5182 .5222 .5252 .532 2.5752 .6142 .6162 .6182 .6242 .6592 .6752 .7382 .7402 .8562 .9172 .9282 .9372 .9372 .9772 .996 3.0303 .1253 .1393 .1453 .2203 .2233 .2353 .2433 .2643 .2723 .2943 .3323 .3463 .3773 .4083 .435 3.4933 .5013 .5373 .5543 .5623 .6283 .8523 .8713 .8863 .9714 .0244 .0274 .2254 .3955 .020

We apply our model as:

1. Check the validity of two parameter negative exponential for given data sets see Figure 2 .

2. Compute maximum penalized likelihood estimator and Bayes estimator of $R$ for complete data. Table 4 shows the results.

3. Compute the maximum penalized likelihood and Bayes estimator for incomplete data and corresponding confidence interval using two different progressively censored samples schemes (Table 5) and data analysis results for incomplete data (Table 6). 

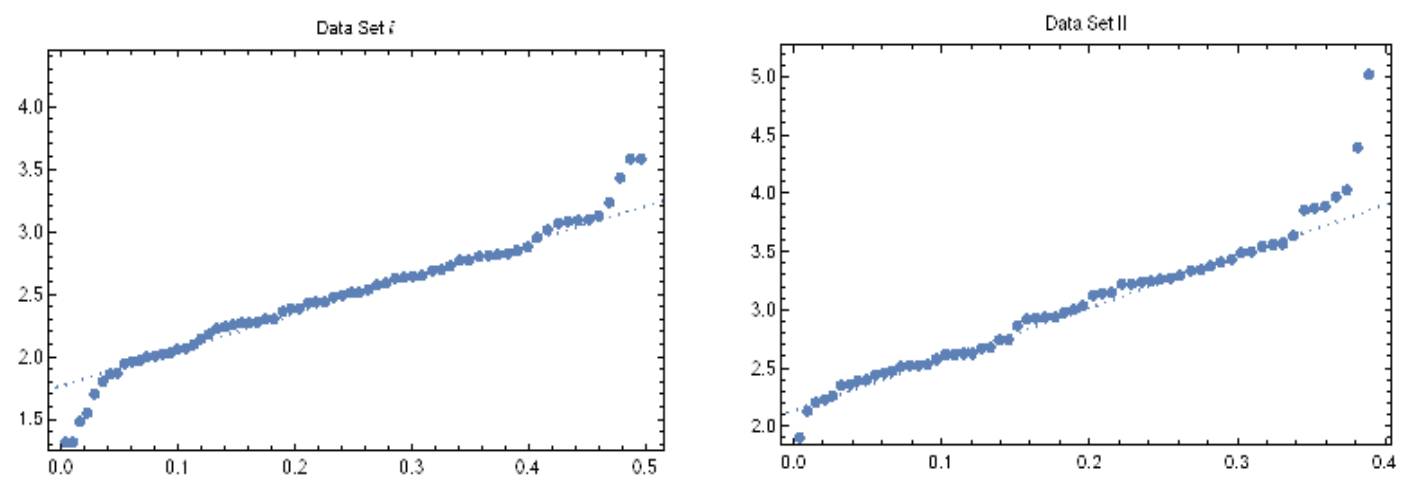

Figure 2: Fitted two parameter negative exponential distribution for data set I and data set II.

Table 4: Data analysis results for complete data

\begin{tabular}{cccc}
\hline \hline$\left(\lambda_{1}^{*}, \lambda_{2}^{*}, \mu_{1}^{*}, \mu_{2}^{*}\right)$ & $\hat{R}^{*}$ & $R^{B}$ & ACI \\
\hline$(1.3707,1.214,1.8792,1.2944)$ & 0.70986 & 0.6807 & $(0.6985,0.7098)$ \\
\hline \hline
\end{tabular}

ACI = asymptotic confidence interval.

Table 5: Data and the corresponding censored schemes

\begin{tabular}{ccccccccccc}
\hline \hline$x_{i}$ & 1.312 & 1.479 & 1.552 & 1.803 & 1.944 & 1.858 & 1.966 & 2.027 & 2.055 & 2.098 \\
$P_{i}$ & 1 & 0 & 1 & 2 & 0 & 0 & 3 & 0 & 1 \\
\hline$y_{j}$ & 1.901 & 2.132 & 2.257 & 2.361 & 2.396 & 2.445 & 2.373 & 2.523 & 2.532 & 2.570 \\
$Q_{j}$ & 0 & 2 & 1 & 0 & 1 & 1 & 2 & 0 & 0 & 44 \\
\hline \hline
\end{tabular}

Table 6: Data analysis results for incomplete data

\begin{tabular}{|c|c|c|}
\hline \multicolumn{3}{|c|}{$\left(\lambda_{1}^{p \prime}, \lambda_{2}^{p \prime}, \mu_{1}^{p \prime}, \mu_{2}^{p \prime}\right)=(2.8849,2.2840,1.257,1.2576)$} \\
\hline Method & Estimator of $R$ & Confidence interval \\
\hline Maximum penalized likelihood & 0.5580 & $(0.5000,0.5800)$ \\
\hline Bayes estimator (prior 1) & 0.5313 & $(0.4793,0.5834)$ \\
\hline Bayes estimator (prior 2) & 0.4756 & $(0.4289,0.5222)$ \\
\hline Bayes estimator (prior 3) & 0.5138 & $(0.4634,0.5641)$ \\
\hline
\end{tabular}

\section{Conclusions}

In this paper, we apply a maximum penalized likelihood method to estimate a stress-strength parameter based on two parameter negative exponential distribution and compare it with the regular maximum likelihood method. Subsequently, it was decided the maximum penalized likelihood method is better than the regular maximum likelihood method because it has a smaller variance. We apply the maximum penalized likelihood method for complete and incomplete data. We also introduce a Bayes estimator using Lindley approximation for complete data and MCMC technique for incomplete data. A simulation study is performed to compere between different estimators in biases and MSEs for complete and incomplete data. The Bayes estimate of $\mathrm{R}$, and the corresponding credible interval can be obtained using the Gibbs sampling technique. In addition, we apply our model on real data and recommend the use of two parameter negative exponential distribution as a reliability model for complete and incomplete data. 


\section{References}

Ali MM, Pal M, and Woo J (2012). Estimation of $P(Y<X)$ in a four-parameter generalized gamma distribution, Austrian Journal of Statistics, 41, 197-210.

Al-Mutairi DK, Ghitany ME, and Kundu D (2013). Inferences on stress-strength reliability from Lindley distributions, Communications in Statistics Theory and Methods, 42, 1443-1463.

Asgharzadeh A, Valiollahi R, and Raqab M (2011). Stress-strength reliability of Weibull distribution based on progressively censored samples, SORT, 35, 103-124.

Badar MG and Priest AM (1982). Statistical aspects of fiber and bundle strength in hybrid composites. In T. Hayashi, K. Kawata, and S. Umekawa (Eds.), Progress in Science and Engineering Composites (pp. 1129-1136), ICCM-IV, Tokyo.

Balakrishnan N and Aggarwala R (2000). Progressive Censoring: Theory, Methods and Applications, Birkhauser, Boston.

Basu AP (1964). Estimates of reliability for some distributions useful in reliability, Technometrics, $\mathbf{6}$, 215-219.

Beg MA (1980). On the estimation of $P(Y<X)$ for two-parameter exponential distribution, Metrika, 27, 29-34.

Birnbaum ZW (1956). On a use of the Mann-Whitney statistic. In Proceedings of Third Berkeley Symposium on Mathematical Statistics and Probability, (Vol. 1, pp. 13-17), University of California Press, CA.

Chen MH and Shao QM (1999). Monte Carlo estimation of Bayesian credible and HPD intervals, Journal of Computational and Graphical Statistics, 8, 69-92.

Downtown F (1973). The estimation of $P(Y<X)$ in the normal case, Technometrics, 15, 551-558.

Enis $\mathrm{P}$ and Geisser S (1971). Estimation of the probability that $P(Y<X)$, Journal of the American Statistical Association, 66, 162-168.

Ghitany ME, Al-Mutairi DK, and Aboukhamseen SM (2015). Estimation of the reliability of a stressstrength system from power Lindley distributions, Communications in Statistics Simulation and Computation, 44, 118-136.

Greco L and Ventura L (2011). Robust inference for the stress-strength reliability, Statistical Papers, 52, 773-788.

Hussian MA (2013). On estimation of stress-strength model for generalized inverted exponential distribution, Journal of Reliability and Statistical Studies, 6, 55-63.

Iwase K (1987). On UMVU estimators of $P(Y<X)$ in the 2-parameter exponential case, Memoirs of the Faculty of Engineering, Hiroshima University, 9, 21-24.

Kelley GD, Kelley JA, and Schucany WR (1976). Efficient estimation of $P(Y<X)$ in the exponential case, Technometrics, 18, 359-360.

Kundu D and Gupta RD (2006). Estimation of $p(Y<X)$ for Weibull distribution, IEEE Transactions on Reliability, 55, 270-280.

Kundu D and Raqab MZ (2009). Estimation of $R=P(Y<X)$ for three-parameter Weibull distribution, Statistics and Probability Letters, 79, 1839-1846.

Krishnamoorthy K, Mukherjee S, and Guo H (2007). Inference on reliability in two- parameter exponential stress-strength model, Metrika, 6, 261-273.

Lindley DV (1980). Approximation Bayesian methods, Trabajos de Estadistica, 21, 223-237.

McCool JI (1991). Inference on $P(X<Y)$ In the Weibull case, Communications in Statistics Simulation and Computation, 20, 129-148.

Najarzadegan H, Babaii S, Rezaei S, and Nadarajah S (2016). Estimation of $P(Y<X)$ for the Lévy 
distribution, Hacettepe Journal of Mathematics and Statistics, 45, 957-972.

Pal M, Ali MM, and Woo J (2005). Estimation and testing of $P(Y>X)$ in two-parameter exponential distributions, Statistics, 39, 415-428.

Raqab MZ and Kundu D (2005). Comparison of different estimators of $P(Y<X)$ for a scaled Burr Type $X$ distribution, Communications in Statistics Simulation and Computation, 34, 465-483.

Rezaei S, Tahmasbi R, and Mahmoodi M (2010). Estimation of $P[Y<X]$ for generalized Pareto distribution, Journal of Statistical Planning and Inference, 140, 480-494.

Saracoglu B, Kinaci I, and Kundu D (2012). On estimation of $R=p(Y<X)$ for exponential distribution under progressive type-II censoring, Journal of Statistical Computation and Simulation, 82, 729-744.

Sathe YS and Shah SP (1981). On estimation $P(Y<X)$ for the exponential distribution, Communications in Statistics and Theory and Methods, 10, 39-47.

Shahsanaei F and Daneshkhah A (2013). Estimation of stress-strength model in generalized linear failure rate distribution, ArXiv:1312:0401v1.

Tong H (1974). A note on the estimation of $P(Y<X)$ in the exponential case, Technometrics, 16, 625.

Tong $\mathrm{H}$ (1977). On the estimation of $P(Y<X)$ for exponential families, IEEE Transactions on Reliability, 26, 54-56.

Wong A (2012). Interval estimation of $P(Y<X)$ for generalized Pareto distribution, Journal of Statistical Planning and Inference, 142, 601-607.

Zheng M (2013). Penalized Maximum Likelihood Estimation of Two-Parameter Exponential Distributions. A Project submitted to the faculty of graduate school of the University of Minnesota. 
